\title{
artículos
}

\section{La orientación vocacional en la UNAM: el caso de bibliotecología}

\author{
Patricia Hernández Salazar
}

\section{RESUMEN}

Una adecuada elección de carrera produce profesionales satisfechos con su formación. Para que esta condición se logre es necesario que los estudiantes interesados en determinada carrera obtengan la mayor información sobre la misma, a través de los servicios de orientación vocacional que ofrecen diversas instituciones.

En este documento se presentan los servicios de orientación vo ca cio nal que ofre ce la Universidad Nacional Autónoma de México, sobre la carrera de Licenciado en Bibliotecología.

En primer lugar se describen los antecedentes de la orientación vocacional en la UNAM, así como las dependencias que realizan esta actividad. Enseguida, se muestran los programas que se siguen en los planteles de la Escuela Nacional Preparatoria y en los del Co le gio de Cien cias y Hu ma ni da des. Pos te rior men te, se des criben y ana li zan los ma te ria les que se usan para orien tar sobre la carrera de Bibliotecología.

Por último, se presenta un análisis de la información utilizada para orien tar sobre laLicenciatura en Bibliotecología,incluyendo algunas consideraciones finales acerca de la conveniencia de dicha información.

\begin{abstract}
An adequate selection of a major field of university study produces graduates who are satisfied with their training. In order to achieve this condition, it is ne ces sary that stu dents in te res ted in a cer tain field ob tain the greatest amount of information regarding that field. This is done through the services of vocational orientation or counseling that diverse institutions offer.

In this document the services of vocational orientation that the Universidad Nacional Autónoma de México offers, concerning the Bachelor program in Library Science, are studied.

First of all, the historical background of the vocational orientation given in the UNAM is presented, as well as the specific agencies that provide such counseling. Next are shown the programs that are followed in the schools of the Escuela Nacional Pre pa ra to ria and the Co le gio de Cien cias y Hu ma ni da des (two types of Me xi can high schools). Then the ma te rials used to provide orientation about the field of Library Science are described and analyzed.

Finally, the information utilized to orient students about the field of a Bachelor's degree in Li brary Scien ce is analyzed. Some final observations are included about the utility of said information.
\end{abstract}

\section{ANTECEDENTES}

El surgimiento de la orientación vocacional en la UNAM data de al gu nas dé ca das atrás. En 1933 se or ga ni za ron las primeras conferencias de contenido profesiográfico, como una señal de interés hacia la orientación profesional.'

Para 1953, como resultado de la primera reunión nacional de la Sociedad de Estudios Profesionales, se concretaron las actividades de orientación en la UNAM, mediante la creación del Departamento de Psicopedagogía y Salud Mental de la UNAM, cu yos ob je ti vos fue ron: in for mar a sus alum nos so bre la institución a la que pertenecían, asesorarlos en sus métodos de estudio y en sus problemas vocacionales y profesionales.
La Fa cul tad de Fi lo so fía y Le tras, como apo yo a la for ma ción de orientadores implantó en 1956 un curso específico dentro del plan de estudios de la carrera de Pedagogía (actualmente vigente). Asimismo, la Facultad de Psicología estableció el Doctorado de Orientación Profesional (ya desaparecido).

En 1964 se re es truc tu ró el plan de es tu dios de la ENP, du rante el rec to ra do del doc tor Ig na cio Chá vez. El nue vo plan es tableció un ciclo de 3 años, los dos primeros for ma dos por materias comunes (humanísticas, técnicas y científicas) y el tercero integrado por materias específicas distribuidas en cinco áreas, entre las cuales el alumno podía seleccionar de acuerdo a la carrera que pretendieraestudiar. Este plan de estudios es el vigente hasta la fecha. 
Dicha reestructuración dio como resultado que el proceso de orientación adquiriera mayor importancia, ya que el estudian te de be ría ele gir su pro fe sión a tra vés de una de ci sión vocacional.

Con base en las re for mas uni ver si ta rias de 1966, en las que se planteó la adecuación de la educación a las necesidades sociales, económicas y políticas del país, la orientación se convirtió en integral: pretendió unificar factores sociales, pedagógicos, culturales, escolares, vocacionales y políticosdelos estudiantes.

Posteriormente se fusionaron el Departamento de Orientación de la ENP y el Departamento de Psicopedagogía,creándose la Dirección General de Orientación y Servicios Sociales (DGOSS), la cual ade más de ofre cer orien ta ción rea li za ba funciones de acción social como otorgamiento de becas y bolsa de trabajo. Desde entonces a la fecha no ha habido gran des cam bios en cuan to a los prin ci pios de la orien ta ción.

Actualmente, los servicios de orientación en la UNAM son otorgados por dos instancias: la Dirección General de Orientación Vocacional(DGOV) y el Departa men to de Psicopedagogía del Colegio de Ciencias y Humanidades $(\mathrm{CCH})$.

\section{Dirección General de Orientación Vocacional}

Esta Dirección derivó de la DGOSS, fue establecida en 1973 como un intento por solucionar problemas de rendimiento escolar, vocacional y de deserción de la población estudiantil.

Su objetivo general es:

"Coadyuvar a la formación integral de los estudiantes universitarios y ex trau niversitarios de los ni veles me dio, [y] medio su pe rior; ofre cién do les in for ma ción so bre las dis tin tas alternativas profesionales que imparten en la UNAM y otras instituciones educativas del país; brindándoles las herramientas para que conozcan sus capacidades y las aptitudes re que ri das para el de sa rro llo de su fu tu ra ocu pa ción; apo yándolos en el tránsito de la adolescencia a una integración madura y conciente [sic] a la sociedad y; asesorándolos en su proceso de aprendizaje y desempeño académico" ${ }^{2}$

El principio de orientación que maneja la DGOV, es el de orientación integral o edu ca ti va, de la que de ri van la es co lar, la vocacional y la profesional.

Para esta dependencia los diferentes tipos de orientación estánín ti ma mente re laciona dos, los servicios de orien ta ción no es tán de li mi ta dos, de tal ma ne ra que lo profe sio nal in volu cra lo vocacional y ambos lo escolar. Los servicios de orientación que ofrece son:

- Estudios vocacionales individuales.

- Infor ma ción so bre el Sis te maEdu ca ti vo Na cio nal en los niveles medio básico, me diosu perior, superior, es pecialidades y posgrados.

- Talleres de técnicas de estudio, elección de carrera y desarrollo integral.

- Ciclo de conferencias.
Estos servicios pue den ser gru pa les, in di vi dua les, por te léfono o por correo.

Los medios que utilizan son: pruebas psicométricas, entrevistas, técnicas grupales, material audiovisual, material bibliográfico, conferencias, exposiciones, etc.

Además ofrece servicios de educación continua dirigidos a los responsables de proporcionar orientación de cualquier institución, mediante la im par ti ción de cur sos y ase so rías relacionadas con la orientación educativa.

\section{De par tamen tode Psi cope da go gía del Cole giode Ciencias y Humanidades}

Después de la creación de los Colegios, en 1971, por el enton ces rec tor Pablo Gon zález Ca sa no va, se crean en 1973 los Departamentos de Psicopedagogía en la Coordinación del $\mathrm{CCH}$ y en cada uno de los planteles.

El objetivo de la orientación vocacional en el CCH es informar al alumno sobre las posibles carreras que existen en la Universidad y en las diversas instituciones a nivel superior y técnico.

Siguiendo el esquema de participación activa, característico del $\mathrm{CCH}$, di cha orien ta ción se lle va a cabo a tra vés del pro pio alumno, haciendo que investigue sobre las características y alternativas profesionales para que sea capaz de elaborar un plan de acción que oriente sus decisiones escolares y vocacionales.

Los me dios son igua les que los uti li za dos por la DGOV, además a ellos se agrega material au to di rigido y téc nicas de análisis como la etnometodología. ${ }^{3} \mathrm{Al}$ igual que en la DGOV, el concepto de orientación es in te gral sin de li mi tar cada uno de sus tipos.

Ambas instancias orientadoras han seguido principalmente, tres modelos de orientación: científico, desarrollistas y clínico. ${ }^{4}$

El mo de lo cien tí fi co está ba sa do en la cla si fi ca ción de los individuos se gún sus ap ti tu des, las cua les son re la cio na das con su futuro profesional, mediante la aplicación de pruebas de aptitudes y habilidades (enfoque testista).

El desarrollista, toma en cuenta una visión sociológica educativa, se pretende ubicar al sujeto en un contexto laboral, bajo la premisa de que a mayor educación mejores oportunida des de em pleo. Este mo de lo uti li za un aba ni co in for ma cional sobre las diversas profesiones existentes en diferentes instituciones educativas (enfoque profesiográfico).

Por úl ti mo, el mo de lo clíni co se basa en la in te gra ción del suje to con su me dio am bien te: so cial, fa mi liar y con sus in te reses y personalidad. Utiliza elementos depsicologíaprofunda paradi ri gir y apo yar la de ci sión del su je to (enfo que clínico). 
El proceso de orientación actual en la UNAM, involucra los tres mo de los, con el fin de ha cer lo más ob je ti vo y que los resultados sean realmente útiles y aplicables.

\section{PROGRAMA DE ORIENTACION DE LA ESCUELA NACIONAL PREPARATORIA}

El programa fue elaborado por la DGOV, como un producto instrumentable para otorgar servicios de orientación a nivel me dio su pe rior, el do cu men to se ti tu la Pro gra ma Ge ne ral Nivel Bachillerato 1987-1988. Este programa es el que está vigente y es desarrollado en cada uno de los 9 planteles de la ENP.

\section{Objetivo}

Desarrollar actividades informativas y formativas, con el fin de apo yar a los alum nos de la ENP en su de sem pe ño es co lar y elección vocacional.

\section{Estructura}

El pro gra ma está con for ma do por tres gran des pro gra mas, dirigidos a cada grado escolar:

- Integración universitaria -4o. grado.

- Orientación vocacional -5o. grado.

- Apoyo a la toma de decisión -6o. grado.

Para aplicar el programa se manejan dos modalidades:

1) Atención grupal:

- Atención directa frente a un grupo.

- Ta lle res de par ti ci pa ción vo lun ta ria a los que pue de asis tir cualquier estudiante sin importar el grado.

- Actividades complementarias: conferencias, mesas redondas, visitas guiadas.

2) Atención individual:

Atención permanente a los estudiantes de cualquier grado, sobre diversas situaciones, escolares, vocacionales, personales y/o familiares.

Los 3 programas enunciados es tán en mar ca dos en la mo da lidad de atención grupal. A continuación se presenta la información general de cada programa.

\section{Programa: Integración Universitaria}

Grado: 4o. año.

Objetivo específico: Motivar en los alumnos un proceso crítico y reflexivo sobre algunos aspectos que facili ten su participación como universitariosa través del conocimiento de la ins ti tu ción, su si tua ción como adoles centes, ac tua ción aca démica y elección ocupacional. ${ }^{5}$

Contenido:

Tema 1. La UNAM: su significado y trascendencia. Tema 2. Aspectos significativos de la adolescencia. Tema 3. Apoyo al aprendizaje.

Tema 4. Introducción a la elección de carrera.

Duración: 17 sesiones de 50 minutos cada una.

\section{Programa: Orientación Vocacional}

Grado: 5o. año.

Objetivo específico: Los alumnos efectuarán su elección de carrera con base en un proceso sistemático de análisis de información laboral, educativa, personal y profesiográfica, sustentada en un modelo de toma de decisión.

Contenido:

Tema: 1. Motivación e introducción al proceso de elección de carrera.

Tema 2. Información profesiográfica.

Tema 3. Evaluación de la información y toma de decisión. Duración: 18 sesiones de 50 minutos cada una.

\section{Programa: Apoyo a la toma de decisión Vocacional}

Grado: 6o. año.

Objetivo específico: Los alumnos adquirirán elementos que apoyen, reafirmen o definan su elección ocupacional. ${ }^{7}$

Contenido

1. Encuesta diagnóstica.

2. Aplicación del cuestionario de reflexión.

3. Discusión de los resultados del cuestionario.

4. Estructuración del taller.

Duración: 11 sesiones de 50 minutos cada una.

Para lle var a cabo el pro gra ma se em plean di fe ren tes téc ni cas didácticas:

- Expositiva, tanto por parte del orientador como de los participantes.

- Lecturas individuales.

- Interrogatorio.

- Sesiones plenarias.

- Dramatizaciones o simulacros.

- Dinámicas grupales: corrillos, Phillip 6/6, etc.

- Visitas guiadas.

Eldocumen toincluye un crono gra ma de ac ti vidades ten ta tivo, el orientador tiene la posibilidad de seguir o no las técnicas y tiempos sugeridos, de acuerdo a su criterio y experiencia.

Para ins tru men tar el pro gra ma exis te en cada plan tel una sección de orientación vocacional, con personal para tal fin. La DGOV fun cio na como co or di na do ra de la ela bo ra ción y aplicación del programa.

\section{Material de Apoyo}

El ma te rial que uti li za el orien ta dor y el que se pro por cio na al estudiante es de los siguientes tipos:

Bibliográfico:

Artículos de revistas. Los orientadores se basan en antologías del Programa General que elabora la DGOV. 
Publicaciones de la DGOV. Folletería, trípticos, Guía de Carreras, etc.

Directorios.

- Batería de pruebas psicométricas.

- Audiovisual:

Videocas settes VHS 1/2 pulgada: generales y profe siográficos por área disciplinaria, poseen en total 62 .

Diaporamas.

Programas de televisión.

Programas de radio.

Banco de datos del Sistema Nacional de Orientación Educativa (SNOE).

A continuación se presentan algunas cifras de servicios de orien ta ción ofre ci dos por la DGOV (en sus ins ta la cio nes o en los planteles de la Escuela Nacional Preparatoria ENP), durante 1989:

\begin{tabular}{|l|r|}
\hline \multicolumn{1}{|c|}{ Servicios de orientación } & $\begin{array}{c}\text { Número de } \\
\text { orientados }\end{array}$ \\
\hline Información profesiográfica & 22,910 \\
\hline Entrevistas & 13,017 \\
\hline Entrevistas individuales & 4,808 \\
\hline Exámenes psicométricos & 3,149 \\
\hline Orientación por correspondencia & 371 \\
\hline Talleres de opción de carrera & 43 \\
\hline
\end{tabular}

PROGRAMA DE ORIENTACION VOCACIONAL DEL COLEGIO DE CIENCIAS Y HUMANIDADES

En los planteles del $\mathrm{CCH}$ no existe un programa impreso (como en el caso de la ENP), cada plantel cuenta con un Departamento de Psicopedagogía y genera sus propios programas.

Por lo anterior, a con ti nua ción se pre sen tain for macióngeneral so bre el proce so de Orien ta ción Vo ca cio nal en los plan teles del Colegio de Ciencias y Humanidades.

\section{Objetivo.}

El objetivo de la orientación en el Colegio de Cien cias y Humanidades es "dar al alumno un panorama amplio de las posibles carreras que existen en la Universidad y las distintas instituciones a nivel superior y técnico".

Si guien doelobje ti vo fun da mental del Cole gio que es for mar alumnos críticos, creativos y responsables, a través de la orientación se pretende que el alumno se informe de las características y alternativas profesionales, que investigue las alternativas que se le pre sen tan, para que sea ca paz de ela borar un plan de acción que le permita tener elementos que orienten sus decisiones futuras.

\section{Estructura.}

Los diferentes programas que conforman el plan de orientación deben presentar una estructura uniforme, la cual comprende las siguientes fases:
1. Ambientación: integración del alumno al sistema.

2. Conocimiento y comprensión de las diferentes materias.

3. Ubicación de los centros educativos.

4. Requisitos administrativos para ingresar.

5. Duración de la carrera.

6. Planes de estudio.

7. Actividades que se realizan en cada carrera y en qué lugares.

8. Horario de estudio.

9. Relación con otras carreras.

10. Aptitudes que se requieren del alumno.

11. Facilidad de trabajar mientras se estudia.

12. Facilidad de encontrar trabajo al egresar.

Dado que la población estudiantil de cada plantel es muy gran de (15,000 alum nos apro xi ma da men te) y que el per so nal que rea li za las ac ti vi da des de orien ta ción es muy poco ( 4 por plantel); la orientación tiende a ser masiva y básicamente infor ma ti va, los me dios de di fu sión que uti li zan en el CCH son:

1. Conferencias impartidas por profesores de diversas Facultades.

2. Conferencias impartidas por profesores del Colegio.

3. Conferencias impartidas por alumnos de diversas Facultades.

4. Conferencias impartidas por alumnos de los Colegios.

5. Mesas redondas.

6. Visitas a los Centros de Trabajo Profesional.

7. Exposiciones en los planteles.

En menor proporción se ofrecen servicios personalizados de OV, como la evaluación de intereses y aptitudes, y el análisis de problemas familiares, entre otros.

\section{Material de apoyo.}

Los Co le gios uti li zan el ma te rial pro du ci do por la DGOV, así comopublicaciones propias de cada DepartamentodePsicopedagogía:

\section{- Bibliográfico:}

- Material autoadministrado (publicado por el Departamento de Psicopedagogía de la Coordinación del $\mathrm{CCH})$.

- Trípticos.

- Boletines informativos mensuales de cada Departamento.

- Carteles.

- Audiovisual:

Diaporamas.

Videocassettes.

Películas. 
- Batería de exámenes psicométricos:

Aplicados y evaluados por el orientador.

Autoaplicables.

Como se puede apreciar la extensión de la información que se ofrece de ambos Programas no es equilibrada (el Programa de la ENP es más extenso), sin embargo, se consideró con ve nien te pre sen tar to dos los da tos ob te ni dos con el fin de describir el panorama real de ambas instituciones.

\section{ORIENTACION VOCACIONAL SOBRE LA CARRE- RA DE BIBLIOTECOLOGIA}

El material que se utiliza para orientar sobre la carrera es:

\section{Tríptico informativo}

LaDGOV cla si fi có las carre ras en 11 cam pos ocu pa cionales:

I. Desarrollo del Agro

II. Salud

III. Industrias Extractivas

IV. Industria de la Construcción

V. Industria de la Transformación y Maquinaria

VI. Economía y Administración

VII. Relaciones Intergrupales

VIII. Investigación

IX. Preservación de la Cultura

X. Expresión Visual

XI. Canto y Música

Según esta clasificación, Bibliotecología queda incluida en el campo IX Preservación de la Cultura:

\section{Objetivo:}

Preservación, incremento y trans mi sión de diversos as pectos de la cultura nacional y universal, tienen a la docencia como actividad principal y realizan diversas formas de investigación. Se orientan en general al desarrollo de la conciencia histórica y cultural entre la población. ${ }^{10}$

Después de analizar los objetivos de cada campo ocupacional, se observó que la carreradeBibliotecología está adecuadamente clasificada, ya que su objetivo principal es organizar y trans mi tir in for ma ción de cual quier tipo, lo que per mi te preservar el acervo cultural tanto nacional como universal.

El tríptico contiene, además del obje ti vo y ca rre ras del gru po IX, los siguientes aspectos:

\section{Licenciado en: Bibliotecología}

1. ¿Qué es y qué hace?

Es el profesional ca paz de pla near, ins tru men tar, or ga ni zary dirigir centros de material hemerográfico, centros de información y documentación, bancos de datos y redes de información automatizados.

\section{Entre sus principales actividades están:}

Seleccionar material documental de acuerdo a las necesidades de los usuarios.

Catalogar y clasificar el material.

Planificar el servicio bibliotecario.

Atender con sul tas y orien tar a los usua rios en la bús que da de información.

Organizar y administrar las bibliotecas y cen tros de in formación.

Docencia e investigación.

\section{3. ¿Dónde realiza su actividad?}

En la aten ción a usua rios y en el de sa rro llo de sis te mas do cumentales, abarca diferentes tipos de bibliotecas: nacionales, universitarias, públicas, especiales, escolares e infantiles.

En instituciones públicas o privadas, centros de investigación, universidades, industrias, hospitales, embajadas, organismos internacionales y/o consorcios mundiales, entre otros. Tam bién pue de co la bo rar en tra ba jos de do cen cia e investigación.

\section{4. ¿Cómo realiza su actividad?}

Para ello utiliza técnicas bibliográficas, documentales y de informática.

\section{Características deseables en el alumno:}

Es con ve nien te que los as pi ran tes a esta ca rre ra cuen ten con:

- Interés por los libros y la preservación de la cultura a través de ellos.

- Buen manejo del lenguaje oral y escrito.

- Gusto por la lectura.

- Ser ordenado y sistemático.

- Tener habilidad para establecer buenas relaciones interpersonales.

- Capacidad de autocrítica y de crítica constructiva.

- Facilidad hacia los idiomas.

- Constanciay tenacidadenlasactividadesemprendidas.

- Ser capaz de trabajar en equipo.

\section{Condiciones específicas relacionadas con el estudio:}

La carga aca dé mi ca exi ge el tiem po comple to deles tu diante, por lo que no podrá trabajar durante sus estudios.

La carrera implica gastos sobre libros, revistas y fotocopias, además de los que pudieran originar algunos viajes de estudio dentro del país.

\section{Plan de Estudios ${ }^{11}$}


8. Otras institucionesque imparten la carrera, ade más de la UNAM:

Escuela Nacionalde Bibliote cono míay Archivono mía, SEP, D.F. (4 años)

Universidad Autónoma de Guadalajara, Jal. (8 semestres)

Universidad de Guadalajara, Jal. (8 semestres)

Universidad Autónoma de San Luis Potosí, S.L.P. (8 semestres) $)^{12}$

Se con si de ra que lo ex pre sa do en los pun tos del 1 al 4 es pertinente, en cuanto al punto 5 (características deseables en el alumno) se debería ampliar la primera característica, no sólo debe tener interés por los libros, sino por cualquier material que permita la preservación de la cultura.

Den tro de las con di ciones es pe cíficas re la cio na das con el estudio (punto 6), se plantea la necesidad de adquirir libros y revistas, lo cual es cierto, sin embargo se puede suplir esta ad qui si ción por la apli ca ción de los apren di za jes de los alumnos, en relación a la recuperación de materiales de apoyo para sus estudios, mediante el uso de bibliotecas y/o centros de información.

Los datos del punto 8 (instituciones que imparten la carrera) están desactualizados, las 2 carreras de las Universidades de Guadalajara han dejado de ser impartidas, y no aparece la licenciatura de la Universidad Autónoma de Nuevo León.

\section{Guía de Carreras de la UNAM.}

Esta guía ofrece información profesiográfica sobre las carreras que se imparten en la UNAM, los datos que presenta sobre Bibliotecología son:

\section{Nombre de la carrera:}

Licenciatura en Bibliotecología

\section{Título que otorga:}

Licenciado en Bibliotecología

\section{Duración de los estudios:}

8 semestres

4. Area a la que pertenece según estructura de la E.N.P: 5 Humanidades Clásicas

\section{Carreras afines según el campo ocupacional:} (ver Anexo)

6. Escuelas y Facultades de la UNAM en que se imparte: Facultad de Filosofía y Letras, C.U.

\section{Definición:}

Igual a la información incluida en el Tríptico

\section{Actividades principales:}

Presenta las mismas que el Tríptico
9. Principales conocimientos precurrentes:

Los referentes al ciclo anterior: el Bachillerato

10. Plan de estudios:

- Número de créditos: 242

- De asignaturas obligatorias: 210

- De asignaturas optativas: 32

- Número de asignaturas: 48

- Obligatorias: 42

- Optativas: 6

\section{Materias principales o definitorias:}

En el estudio de la carrera se consideran básicas: teoría bibliotecológica, servicios técnicos (manuales y automatizados), sistemas y servicios de información y bibliografía. La práctica dirigida en el transcurso de la carrera es básica para la formación del profesional.

\section{Inversiones:}

Ver punto 6 del Tríptico

13. Servicios que ofrece la facultad o escuela al estudiante: Se cuenta con biblioteca, fotocopiado, becas, talleres de redacción y traducción.

\section{Perfil personal:}

Tener habilidad para:

- Resolver problemas específicos en forma práctica.

- Manejar personal.

- La crítica constructiva

Tener interés por:

- Estar al día en las nuevas técnicas, estudios, descubrimientos, etc., que le permitan de sa rro llar en me jor for ma su profesión.

- Poner en práctica los conocimientos adquiridos.

Tener facilidad para:

- Analizar

- Sintetizar

- Escribir correctamente

Tener capacidad para

- Razonar ordenadamente

- Tomar decisiones

Tener conocimientos de:

- Una lengua extranjera (mínimo traducción)

Tener el conocimiento de:

- La comunidad en que se va a trabajar y de sus necesidades

Tener:

- Inventiva y creatividad

- Hábito por la lectura ${ }^{13}$

15. Otras instituciones que imparten la carrera: Aparecen las mismas que en el Tríptico. 
Como se puede observar, el Tríptico contiene los datos principales de la Guía de Carreras, los datos complementarios son factuales, por lo que no se analizaron.

\section{Videocassette}

Videocassette con información profesiográficatitulado"Lic. en Bibliotecología", con una duración de 15 minutos.

\section{Exámenes psicométricos generales}

Tanto la DGOV como el Departamento de Psicopedagogía aplican exámenes psicométricos con el fin de determinar un perfil aproximado del estudiante y sugerirle posibilidades profesionales.

Las carac te rís ti cas que mi den en el es tu dian te me dian te es tos exámenes, son:

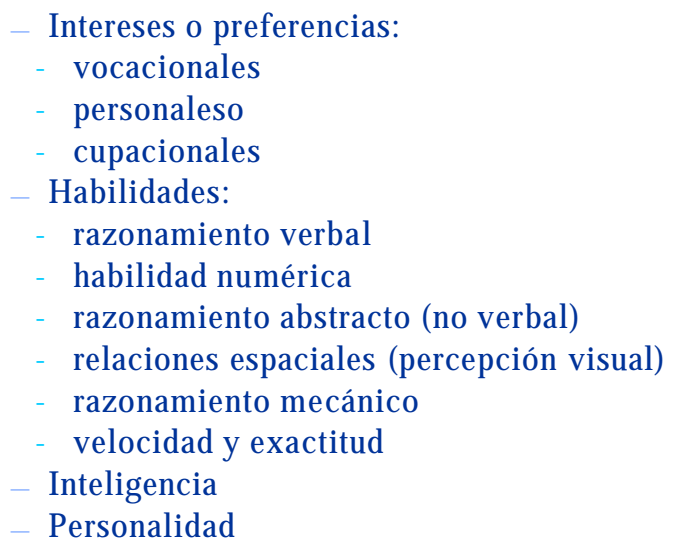

Exis te una po lí ti ca fle xi ble en cuan to a la apli ca ción de exá menes, cada orientador utiliza los que cree más convenientes.

Noexis ten exámenes di se ña dos es pe cial men te para la población universitaria, se les aplica los normalizados internacionalmente.

La interpretación de los perfiles que arroja la psicometría es por áreas más que por ca rre ras es pe cífi cas. Por ejem plo, en la escala de preferencias de Kuder se clasifican las actividades en 10 áreas o intereses:

0 trabajo al aire libre

1 mecánico

2 para el cálculo

3 científico

4 persuasivo

5 artístico-plástico

6 literario

7 musical

8 servicio social

9 trabajo de oficina ${ }^{14}$

De pen dien do delárea don de se ob ten gan los por cen tajes más altos será el perfil de interés del orientado; la carrera de $\mathrm{Bi}$ bliotecología está clasificada en las áreas 6, 8 y 9, de tal ma- nera que si un estudiante está interesado en ella deberá obtener porcentajes altos por lo menos en alguna de ellas.

Asimismo, la tabla de interpretación determina que los interesadosdeberán te ner pre fe ren cia por si tua cio nes no con flicti vas, con ten den cia a for mar se gru pos profe sio na les fe me ninos.

Con las interpretaciones de los diferentes tipos de exámenes se determina el perfil del estudiante, ofreciéndole posteriormente información de las carreras que estén incluidas en el mismo.

\section{Perfil propor cio na do por el Sis te ma Na cional de Orien ta- ción Educativa (SNOE)}

El SNOE fue creado por acuer do pre si den cial el 3 de oc tu bre de 1984 , cuyo objetivo es "... posibilitar a los estudiantesuna elección adecuada de las opciones educativas y laborales futuras mediante la promoción, coordinación y difusión de los diversos elementos que inciden en dicha elección.",

Funciona como un órgano público: nacional, normativo, prioritario, permanente, descentralizado y diferenciado por niveles, tendiente a coordinar la orientación en el Sistema Edu ca ti vo Na cio nal de acuer do al de sa rro llo so cial del país. ${ }^{16}$

Den tro de las he rra mien tas del SNOE exis te una Base de Datos automatizada que proporciona información profesiográfica sobre las carreras que pueden estudiarse en México, los datos que incluye son: nivel, servicio, área, rama, nombre de la carrera, entidad(es) que la imparte(n), plan de estudios, sector al que pertenece.

La DGOV cuenta con dicha base de datos para proporcionar información a los estudiantes interesados en la carrera de $\mathrm{Bi}$ bliotecología.

Presenta la misma información aunque la entidad varíe; si bien debería incluir los planes de estudio, al solicitarlos aparece el mensaje que no es tán da dos de alta. A con ti nua ción se presentan los datos principales del perfil del SNOE:

1. Nivel:

Superior

2. Servicio:

Licenciatura

3. Area:

Ciencias Sociales y Administrativas

4. Rama:

Archivonomía y Biblioteconomía

5. Carrera:

Bibliotecología

6. Entidad:

Varía de acuerdo al perfil solicitado 
7. Información:

Sector productivo, datos económicos

\section{Sector:}

Servicios comu nales, socia les y rama servicios deedu cación.

9. Duración:

Ocho semestres en la República Mexicana.

\section{Saturación:}

Esta es una pro fe sión con alto ín di ce de sa tu ra ción en to das las instituciones del país.

\section{Perfil:}

In clu ye la mis ma in for ma ción del Tríp ti co de DGOV en cuanto a qué es y qué hace.

\section{Servicios de Educación:}

Comprende los establecimientos públicos y privados dedicados a proporcionar servicios edu ca ti vos de todo tipo: jar dines deniños, primarias, secundarias, preparatorias, vocacionales, universidades, escuelas comerciales, de idiomas, técnicas, de baile, de arte, de adiestramiento deportivo. Así como centros de in ves ti ga ción cien tífi ca y tec no ló gi ca. Los sis te mas de edur cación abier ta y los servi cios de di fu sión cul tu ra les [sic], ta les como bibliotecas, museos, etc.

\section{Expectativas de la rama económica:}

Dependerá principalmente de la acción del Estado en materia de educación. Sin embargo, se espera una importante evolución de los servi cios edu ca ti vos im parti dos por el sec tor privado.

14. Instituciones que imparten la carrera:

Universidad Nacional Autónoma de México, D.F.; Escuela Nacional de Archivonomía y Biblioteconomía, D.F.; Universidad Autónoma de Nuevo León, Monterrey, N.L.; Universidad Autónoma de Guadalajara, Jal. ${ }^{17}$

La profesiografía del SNOE está incompleta, en esta base de datos la carrera de Bibliotecología ha sido clasificada en la rama de Servicios Educativos, circunscribiéndola a servir como apoyo educativo solamente.

En el punto 10 se expresa que la profesión está altamentesaturada, sin aclarar que la saturación se refiere al número de pla zas que ofre cen las ins ti tu cio nes que im par ten la ca rre ra, y no a la sa tu ra ción de profe sio na les en el merca do de tra bajo.

Más aún, las Es cue las de Bi blio te co lo gía ge ne ral men te ofrecen un cupo reducido, aproximadamente reclutan como número máximo 80 estudiantes anualmente.

La información de los puntos 12 (servicios de educación) y 13 (ex pec ta ti va de la rama eco nó mi ca) es in com ple ta, pues la profesión también apoya a los sectores industrial y de servicios.
Al igual que en el per fil de la DGOV, en lo que res pec ta a las ins ti tu ciones queim parten Biblio te co logía (pun to 14), el perfil del SNOE incluía a la Uni versi dad Au tóno ma de Guadalajara, debiéndola dejar fuera debido a que fue suspendida la carrera por falta de planta estudiantil.

El procedimiento para obtener la información contenida en estos materiales, es el siguiente.

Para el tríp ti co y el per fil de la guía de ca rre ra, la DGOV so licita al director de la Facultad de Filosofía y Letras los datos necesarios. Cada coordinador de carrera, en este caso el de Bibliotecología, redacta y envía los que considerarelevantes para su difusión.

La DGOV no tiene ingerencia sobre los contenidos de los materiales, funciona como compiladora, sin modificar la infor ma ción que les es en via da, por par te de la Co or di na ción de la carrera.

Con respecto al videocassette, la DGOV diseña y elaboralos guiones y productos finales con base en la información proporcionada por la Coordinación de Bibliotecología.

El perfil incluido en la base de datos del SNOE fue diseñado por la Secretaría de Educación Pública.

\section{Conferencias, visitas guiadas}

Ade más del ma te rial im pre so y au dio vi sual, la orien ta ción se ofrece a través de conferencias y visitas guiadas al Colegio de Bibliotecología o a diversas bibliotecas.

\section{ANALISIS DE LA INFORMACION UTILIZADA PARA ORIENTAR SOBRE LA LICENCIATURA EN BIBLIOTECOLOGIA}

\section{Perfil DGOV.}

El proceso de obtención de la información que se incluye en el ma te rial de orien ta ción de la DGOV, mues tra que per so nal relacionado con la carreradeBibliotecología participa directa men te en la ela bo ra ción del per fil profe sio gráfi co que se difunde de la misma, de lo que se desprende que es confiable.

Como se pue de ob servar, se han ana li za do al gu nos pun tos incluidos en el perfil DGOV, sin embargo se consideróimportante profundizar sobre su validez, por lo que se pensó en compararlo con alguno "oficial" emitido por profesionales de la Bibliotecología.

Sobre este punto, la Asociación Mexicana de Bibliotecarios, A.C., (AMBAC) organizó la Mesa Redonda sobre Formación de Recursos Humanos para las Bibliotecas con el fin de "Lograr consenso en cuanto a la formación de recursos humanos bajo los siguientes rubros: 1. Perfil profesional; 2. Profesorado; 3. Alumnado; 4. Servicio de apoyo ..."18 
Dicha mesa redonda reunió a directores de escuelas de bibliotecología, coordinadores de programas de maestrías, repre sentan tes delCole gio Na cional de Biblio te carios, y profesores, por lo que las recomendaciones emitidas se tomaron como parámetros de comparación válidos para este análisis.

En el si guien te cua dro se pre sen ta la re la ción en tre los ob je tivos y actividades contenidos en cada per fil, dado que el de la AMBAC es mucho más amplio, se repiten actividades del perfil DGOV, consideradas como equivalentes:

\section{Relación entre los perfiles de AMBAC y DGOV}

\begin{tabular}{|c|c|}
\hline $\begin{array}{c}\text { Perfil AMBAC } \\
\text { OBJETIVO }\end{array}$ & $\begin{array}{l}\text { Perfil DGOV } \\
\text { OBJETIVO }\end{array}$ \\
\hline $\begin{array}{l}\text { For mar pro fe sio na les ca pa ces de: } \\
\text { 1. Se lec cio nar, ad qui rir, or ga ni zar, re cu pe rary di se mi nar } \\
\text { lain formación. } \\
\text { 2. Orien tar al usua rio en la uti li za ción de los re cur sos y } \\
\text { servicios biblio tecarios. } \\
\text { 3. Co no cer la in fraes truc tu ra de fuen tes y re cur sos de in- } \\
\text { formación exis tentes. } \\
\text { 4. Pla ni fi car, or ga ni zar, di ri gir, con tro lar y eva luar los } \\
\text { ser vi cios bi blio te ca rios y de in for ma ción, así como los re- } \\
\text { cur sos fi nan cie ros, hu ma nos y ma te ria les que ha cen po si- } \\
\text { ble la ope ra ción de uni da des de in for ma ción. }\end{array}$ & $\begin{array}{l}\text { Este pro fe sio nal ca- } \\
\text { paz de pla near, ins tru- } \\
\text { mentar, organizary } \\
\text { dirigirbibliotecas, } \\
\text { cen tros de in for ma- } \\
\text { ción y do cu men ta- } \\
\text { ción; ban cos de da tos } \\
\text { y re des de in for ma- } \\
\text { ciónautomatizada. }\end{array}$ \\
\hline $\begin{array}{l}\text { 5. Apli car la téc ni ca de co mu ni ca ción in ter per so nal, de } \\
\text { gru po y de masa. }\end{array}$ & $\begin{array}{l}\text { Tenerhabilidad e in- } \\
\text { te rés paraes tablecer } \\
\text { buenasrelaciones per- } \\
\text { sonales. }\end{array}$ \\
\hline $\begin{array}{l}\text { Perfil AMBAC } \\
\text { ACTINIDADES }\end{array}$ & $\begin{array}{c}\text { Perfil DGOV } \\
\text { ACTIIDADES }\end{array}$ \\
\hline $\begin{array}{l}\text { 1. De ci dir acer ca de las po líti cas de se lec ción y ad qui si- } \\
\text { ción de los materialesdocumentales que de man denlos } \\
\text { usuarios. }\end{array}$ & $\begin{array}{l}\text { 1. Se lec cio na el ma te- } \\
\text { rial do cu men tal de } \\
\text { acuer do a las ne ce si- } \\
\text { dades de los usuarios. }\end{array}$ \\
\hline $\begin{array}{l}\text { 2. Es co ger y apli car las nor mas y re glas más ade cua das } \\
\text { par la ca ta lo ga ción y cla si fi ca ción de los ma te ria les in for- } \\
\text { mativos. }\end{array}$ & $\begin{array}{l}\text { 2. Ca ta lo ga y cla si fi ca } \\
\text { el material, dándole } \\
\text { una organizaciónque } \\
\text { lo haga accesible. }\end{array}$ \\
\hline 3. Ana li zar los ma te ria les do cu men ta les que se ad quie ren. & - Actividad 2 \\
\hline 4. Planificary realizarin vestigaciones bibliográficas. & $\begin{array}{l}\text { 3. Pla ni fi car el ser vi } \\
\text { ciobibliotecario. }\end{array}$ \\
\hline
\end{tabular}

De lo an te rior se des pren de que el per fil de la DGOV es ade cuado, aunque podría ampliarse incluyendo las actividades de la AMBAC que no tienen correspondencia en el cuadro anterior.

\section{Perfil SNOE}

Después de analizar el perfil del SNOE se llegó a la conclusión que no era posible compararlo con las recomendaciones de la AM B AC, ya que des de el ob je ti vo es di fe ren te, no des glo sa actividades y no pre sen ta plan de es tu dios. Este per fil más que informar, desinforma al orientado.

De lo an te rior, ca bría la si guien te in te rro gan te ¿c uál es el pro pósito de la DGOV al proporcionar dicha información, si difiere

\begin{tabular}{|c|c|}
\hline $\begin{array}{l}\text { Perfil AMBAC } \\
\text { ACTIIDADES }\end{array}$ & $\begin{array}{l}\text { Perfil DGOV } \\
\text { ACTIIDADES }\end{array}$ \\
\hline $\begin{array}{l}\text { 5. Au xi liar a los usua rios en la lo ca li za ci'on de la in for- } \\
\text { mación. }\end{array}$ & $\begin{array}{l}\text { 4. Atien de las con sul- } \\
\text { tas de usua rios y los } \\
\text { irien ta en el em pleo } \\
\text { de los ma te ria les y en } \\
\text { la bús que da de in for- } \\
\text { mación. }\end{array}$ \\
\hline $\begin{array}{l}\text { 6. Orien tar y guiar a los usa rios en la con sul ta y ma ne jo } \\
\text { de los materiales. }\end{array}$ & - Actividad 4 \\
\hline $\begin{array}{l}\text { 7. Ela bo rar los ma nua les y re gla men tos ne ce sa rios para el } \\
\text { funcionamien to de los servicios. }\end{array}$ & - Actividad 3. \\
\hline $\begin{array}{l}\text { 8. De ter mi nar la apli ca ción y dis tri bu ción del pre su pues to } \\
\text { a la uni dad a su car go. }\end{array}$ & -Actividad 3. \\
\hline $\begin{array}{l}\text { 9. Participardirectamente en la pla ni fi ca ción de la cons- } \\
\text { truc ción y/o adap ta ción de lo ca les de la uni dad de in for- } \\
\text { mación de sures pon sabi li dad. }\end{array}$ & - Actividad 3. \\
\hline $\begin{array}{l}\text { 10. Pla ni fi car y re or ga ni zar la dis tri bu ciòn de los ma te ria } \\
\text { les do cu men ta les, equi po y es pa cios. }\end{array}$ & - Ac ti vi dad 3 \\
\hline $\begin{array}{l}\text { 11. Pla ne ra y de sa rro llar los ser vi cios de ex ten sión uni } \\
\text { versitaria. } \\
\text { 12. Ela bo rar re sú me nes de los ma te ria les bi blio gráfi cos y } \\
\text { documentales. } \\
\text { 13. Ma ne jar y co no cer los me dios au dio vi sua les y de co- } \\
\text { municación que se emplean en las unidades de in for ma- } \\
\text { ción. } \\
\text { 14. Se lec cio nar los re cur sos hu ma nos ne ce sa rios para el } \\
\text { desarrollo de los servicios bibliotecarios, de información } \\
\text { y documentación. } \\
\text { 15. Par ti ci par en la es truc tu ra ción del si te ma de re mu ne- } \\
\text { raciones, pres ta cio nes e in cen ti vos del per so nal de la uni } \\
\text { dad de in for ma ción. } \\
\text { 16. Ase so rar den tro de su cam po de es pe cia li za ción a par- } \\
\text { ticu lares e ins ti tu ciones que lo soli ci ten. } \\
\text { 17. In ter pre tar las es ta dís ti cas de proce sos y ser vi cios } \\
\text { para coad yu var a la eva lua ción cons tan te de la uni dad a } \\
\text { su car go. } \\
\text { 18. Di se ñar una políti ca de re la cionesin ter de par ta men ta- } \\
\text { les. } \\
\text { 19. Im par tir las ma te rias téc ni cas de la es pe cia li dad, tan to } \\
\text { a los alum nos del ni vel téc ni co y de li cen cia tu ra, así } \\
\text { como en cur sos de ca pa ci ta ción. } \\
20 \text {. Rea li zar la pro mo ción y la pu bli ci dad ne ce sa rias para } \\
\text { dar a co no cer los ser vi cios bi blio te ca rios, de in for ma ción } \\
\text { y documenatción. }\end{array}$ & - Actividad 3. \\
\hline
\end{tabular}

de la elaborada por ellos mismos? ¿no sería más conveniente prescindir de la base de datos del SNOE?

\section{CONSIDERACIONES FINALES}

La DGOV utiliza herramientas psicométricas con el fin de determinar el perfil preliminar del estudiante, para orientarlo hacia las carreras en las que presente mayores posibilidades.La interpretación psicométrica es por áreas y no por carreras. Bibliotecología está incluida en las áreas literaria, de servicio social y trabajo de oficina.

Laco or di na ción de Biblio te cologíain ter vie ne en la ela bo ración del contenido profesiográfico del material promocional de la carrera, publicado por DGOV. 
La información contenida en el material utilizado por la DGOV para ofrecer orientación sobre la carrera de Bibliotecología es confiable, de acuer do a las re co men da cio nes de la I Mesa Redonda sobre Formación de Recursos Humanos para las Bibliotecas, organizada por la AMBAC.

Es re co men da ble am pliar el per fil uti li za do por la DGOV basándose en las recomendaciones de la AMBAC.

Sería con ve nien te la in terven ción de profe siona les de Bibliotecología en la orientación proporcionada en preparatorias y colegios de ciencias y humanidades como por ejemplo, con: conferencias, seminarios, mesas redondas, etc.

En cuan to al per fil del SNOE exis ten dos op cio nes: adap tar lo al de la DGOV o no utilizarlo, para evitar confusiones en el estudiante.

No está desarrolladoel mercado de trabajoen am bos perfiles (DGOV, SNOE), por lo que se hace necesario em pe zar a trabajar en ello.

\section{CITAS BIBLIOGRAFICAS}

1. García Ra mírez, Ita lia. "De sa rrol lo de la orien ta ción en Mé xi co”. En: Congresolatinoamericano de orientación: Memoria. México: UNAM, Dirección General de Orientación y Servicios Sociales, 1970. p. 60

2. UniversidadNacional Autónoma de México. Direc ción Generalde In tercambio Aca démico. $C a$ tálogo de posibilidades de colaboración de la UNAM en el ámbito nacional. México : UNAM, Dirección General de Intercambio Académico, 1987. p. 1

3. Tér mi no ma ne ja do en el Dep to. de Psi co pe da go gía delCCH, que sig ni fi ca ofre cer orien ta ción de acuerdo a las características sociales de los alumnos.

4. Cortés, Francisco. Orientación vocacional. Ponencia al foro local del Congreso Universitario. UNAM. (Documento mecanografiado)

5. Universidad Nacional Au tón no ma de México. Direc ción Generalde Orienta ción Voca cional. Programa general nivel bachillerato 1987- 1988. México : UNAM, Dirección General de Orientación Vocacional, 1988. p. 13.

6. Idem. p. 70

7. UniversidadNacionalAutónoma de México.Dirección Ge neralde Orientación Vocacional $O p$. Cit. p. 127.

8. Es tra da Mar tínez, Gua da lu pe; et. al. "La orien ta ción vo ca cio nal en elCCH”. En: IVSe mina rio de Pedagogía. México : UNAM, Colegio de Ciencias y Humanidades, 1983. p. 7

9. Idem p. 18.

10. UniversidadNacional Au tóno ma de México. Direc ción Ge neralde Orientación Voca cional. Carreras que se imparten en la UNAM. Tríptico informativo.

11. No se con si de ró con ve nien te in cluir el des glo se del Plan de Es tu dios, para su con sul ta re mi tir se a la Guía de Carreras o al Tríptico informativo.

12. Universidad Nacional Autónoma de México. Dirección General de Orientación Vocacional. Orientación Educativa. Tríptico informativo.

13. UniversidadNacional Au tónomade México. Direc ción Generalde Orientación Vocacional. Guía de Carreras 1988. México : UNAM, Dirección General de Orientación Vocacional, 1988. s.p.

14. Woyno, Wladimiro ; Oñoro Ama dor, Raúl E. Kuder :escalade prefe ren ciasforma CHvocacional. Colombia : Ediciones Pedagógicas Latinoamericanas, 1981. pp. 20-21. 
15. Secre taría de Edu ca ción Pública.Pro gramaNacionalde Orientación Edu cativa 1989-1994. México : SEP, 1988. p. 1

16. Secretaría de Educación Pública. Idem p. 2

17. Sis te ma Nacional de Orien ta ción Edu ca ti va. Base de Da tos. Hojaim pre sa del re sul ta do de la búsqueda, 1991.

18. Mesa Re don da so bre For ma ción de Re cur sos Hu ma nos. (1a. : 1980 : Gua na jua to, Gto.). Mesa redonda sobreformación de recur sos humanosparalas bibliotecas. Mé xi co: AM B AC, 1980. p. 10

19. Carac terís tica deriva da del perfil per sonal, no de los obje ti vos, pero que guar da re la ción con el objetivo 5 de la AMBAC

\section{OBRAS CONSULTADAS}

Anastasi, Anne. Psychological testing. New York : Macmillan Company, 1963.

Asocia ción Mexicana de Profe sionales de la Orien tación.Vocabulario 1982-1983. Méxi co, 1984.

Bohoslavsky, Rodolfo. Orientaciónvocacional:laestrategiaclínica. Bue nos Ai res : Nue va Visión, 1976.

Bordas, M.D. Elección de carreras y profesión. Barcelona : Oikos, Tau., 1980.

ColegioNacionaldeBibliotecarios(México). Recomendacionessobrecapacitacióndelpersonal bibliotecarionoprofe sionalylis tade actividadesprofesionales ynoprofesionales. Mé xi co : El Colegio, 1982. $16 \mathrm{~h}$.

Cortés, Francisco. Orientación vocacional. Ponencia al Foro del Congreso Universitario (documento mecanografiado). 1990.

Es tra da Martínez, Gua da lu pe. "La orien ta ción vo ca cio nal en elCCH”. En:IVSemina rio de Psicopedagogía. pp. 4-20. México : UNAM, Unidad Académica del Ciclo de Bachillerato del $\mathrm{CCH}, 1983$.

Hoyos Medina, Carlos Angel. "La orientación educativa : un programa pú bli co de in terés privado” En: Crítica. pp. 41-49. Puebla : Universidad Autónoma de Puebla, 1987

Mesa Re don da so bre For ma ción de Re cur sos Hu ma nos. (1a. : 1980 : Gua najua to, Gto.). Mesa redondasobreformaciónderecursoshumanosparalasbibliotecas. Mé xi co: AM B AC, 1980. $32 \mathrm{p}$.

- - - - (2a. : 1981 : Oaxtepec, Mor.). II Mesa redonda sobre formación de recursos humanos para las bibliotecas : licenciatura. México : AMBAC, 1981. Pág. varia.

- - - - (5a. : 1990 : Mon te rrey, N.L.). V Mesa redon da sobreforma ciónde recur sos humanos: la en se ñan za de la bi blio te co lo gía en Méxi co, niveles de licencia tu raymaes tría, pla nes de estudio vigentes y modalidades de titulación. Documento mecanografiado.

Pous, Raúl; Ed mun do Es co bary Au rea Va re la.Obje ti vos y planes de es tudio de la Es cue la NacionalPreparatoria. Méxi co: UNAM, Direc ción Ge ne ral de la Es cue la Nacional Pre para to ria, 1982.

Se cre taría de Edu ca ción Pública.Pro grama Nacionalde OrientaciónEducativa 1989-1994. México : SEP, 1988. 49 p. 
UniversidadNacional Autónoma de México. Direc ción Generalde Intercambio Aca démico. $C a$ tálogo de posibilidades de colaboración de la UNAM en el ámbito nacional. México, UNAM, DGIA, 1987. Pág. varia.

-----Di rec ción Ge ne ral de Orien ta ción Vo ca cio nal. Guía de Ca rre ras 1988. Mé xi co : UNAM, DGOV, 1988. s.p.

- - - - Dirección General de Orientación Vocacional. Programa general nivel bachillerato 1987-1988. México : UNAM, DGOV, 1988.

Wo yno, Wla di mi ro; Oño ro Ama dor, Raúl.Kuder: es caladepreferenciasforma CHvocacional. Colombia : Ediciones Pedagógicas Latinoamericanas ; 1981. 60 p. 
ANEXO

\begin{tabular}{|c|c|}
\hline CAMPO OCUPACIONAL & $C A R R E R A$ \\
\hline $\begin{array}{l}\text { DESARROLLO DEL AGRO } \\
\text { Desarrollo de la agricultura ganadera, avicultura, psicicultura y silvicultura } \\
\text { se orientan en general a la solución del proglema alimentario. }\end{array}$ & $\begin{array}{l}\text { Biólogo } 2 \\
\text { Ingeniero agrícola } 1 \text { y } 2 \\
\text { Ingeniero en alimentos } \\
\text { Médico veterinario zootecnista } \\
\text { Planificación para el desarrollo agropecuario }\end{array}$ \\
\hline $\begin{array}{l}\text { SALUD } \\
\text { Prevención, diagnóstico y tratamiento de enfermedades, así como rehabili- } \\
\text { tación de pacientes. Se orientan en general a la recuperación y manteni- } \\
\text { miento de la salud en una concepción integral de ésta. }\end{array}$ & $\begin{array}{l}\text { C irujano dentista } \\
\text { Enfermería y obstetricia } \\
\text { Investigación biomédica básica } \\
\text { Médico cirujano } \\
\text { Psicología } \\
\text { Químico farmaceútico biólogo } \\
\end{array}$ \\
\hline $\begin{array}{l}\text { IN DUSTRIAS EXTRACTIVAS } \\
\text { Exploración, descubrimiento, extracción y/o transformación de las riquezas } \\
\text { del subsuelo. Se orientan en general a la obtención de materias primas de } \\
\text { origen mineral. }\end{array}$ & $\begin{array}{l}\text { Ingeniero de minas y metalurgistaI } \\
\text { ngeniero geofísico } \\
\text { Ingeniero geólogo } \\
\text { Ingeniero petrolero } \\
\text { Ingeniero químico } \\
\text { Ingeniero químico metalúrgico } \\
\text { Químico }\end{array}$ \\
\hline $\begin{array}{l}\text { IN DUSTRIA DE LA CONSTRUCCION } \\
\text { Diseño y construcción de habitaciones, hospitales, fábricas, centros co- } \\
\text { merciales y otras obras de infraestructura, realizando y/o tomando en } \\
\text { cuenta el análisis y evaluación de la característica del suelo, su topografía } \\
\text { y leyes que lo rigen. Se orientan en general a la generación de espacios } \\
\text { seguros y confortables para la colectividad. }\end{array}$ & $\begin{array}{l}\text { Arquitecto } \\
\text { Arquitectura del paisaje } \\
\text { Geografía } \\
\text { Ingeniero civil } \\
\text { Ingeniero geofísico } \\
\text { Ingeniero topógrafo y geodesta } \\
\text { Urbanismo }\end{array}$ \\
\hline $\begin{array}{l}\text { IN DUSTRIA DE LA TRANSFORMACION Y MAQU INARIA } \\
\text { Diseño, construcción, adaptación y o mantenimiento de maquinaria, equi- } \\
\text { pos, instrumentos o procesos para la transformación de la materia en } \\
\text { energía y en bienes. Se orientan en general al mantenimiento y perfeccio- } \\
\text { namiento del instrumental y maquinaria de la industria. }\end{array}$ & $\begin{array}{l}\text { Diseño industrial } \\
\text { Físico } \\
\text { Ingeniero civil } \\
\text { Ingeniero en computación } \\
\text { Ingeniero mecánico electricista } \\
\text { Ingeniero químico }\end{array}$ \\
\hline $\begin{array}{l}\text { ECONOMIA Y ADM INISTRACION } \\
\text { Participación en el proceso económico, administrativo público y privado, } \\
\text { que incluye desde el funcionamiento de las áreas de operación como son } \\
\text { las empresas hasta los procesos globales de crecimiento, inversión, co- } \\
\text { mercio, empleo y distribución del ingreso. Se orientan en general al con- } \\
\text { trol, aprovechamiento y optimización de los recursos financieros, materia- } \\
\text { les y humanos. }\end{array}$ & $\begin{array}{l}\text { Actuario } \\
\text { Administración } \\
\text { C iencias políticas y administración pública } \\
\text { Contaduría } \\
\text { Economía } \\
\text { Informática } \\
\text { Matemáticas aplicadas y computación } \\
\end{array}$ \\
\hline $\begin{array}{l}\text { RELACIONES INTERGRUPALES } \\
\text { Explicación a los afectados de los fenómenos sociales y las condiciones } \\
\text { en que ocurren, proporción de formas de solución en casos conflictivos y } \\
\text { generación de formas de aprovechamiento del conocimiento de las leyes } \\
\text { sociales. Se orientan en general al desarrollo y transformación de las rela- } \\
\text { ciones sociales humanas. }\end{array}$ & $\begin{array}{l}\text { Ciencias de la comunicación } \\
\text { Periodismo y comunicación colectiva } \\
\text { Ciencias políticas y administración pública } \\
\text { Derecho } \\
\text { Pedagogía } \\
\text { Psicología } \\
\text { Relaciones internacionales } \\
\text { Trabajo social } \\
\text { Sociología }\end{array}$ \\
\hline
\end{tabular}




\begin{tabular}{|c|c|}
\hline CAMPO OCUPACIONAL & $C A R R E R A$ \\
\hline $\begin{array}{l}\text { IN VEST IG A C ION } \\
\text { Generación de teorías científicas y conocimientos que sirvan de base para } \\
\text { el desarrollo de tecnologías. Se orientan en general al estudio de los fenó- } \\
\text { menos naturales y/o exactos. }\end{array}$ & $\begin{array}{l}\text { Biólogo } \\
\text { Físico } \\
\text { Investigación económica básica } \\
\text { Matemático } \\
\text { Químico } \\
\end{array}$ \\
\hline $\begin{array}{l}\text { PRESERVACION DE LA CULTURA } \\
\text { Preservación, incremento y transmisión de diversos aspectos de la cultura } \\
\text { nacional y universal, tienen a la docencia como actividad principal y reali- } \\
\text { zan diversas formas de investigación. Se orientan en general al desarrollo } \\
\text { de la conciencia histórica y cultural entre la población. }\end{array}$ & $\begin{array}{l}\text { Bibliotecología } \\
\text { Enseñanza de inglés } \\
\text { Estudios latinoamericanos } \\
\text { Filosofía } \\
\text { Geografía } \\
\text { Historia } \\
\text { Lengua y literatura hispánicas } \\
\text { Lengua y literatura modernas alemanas } \\
\text { Lengua y literatura modernas francesas } \\
\text { Lengua y literatura modernas inglesas } \\
\text { Lengua y literatura modernas italianas } \\
\text { Letras clásicas } \\
\text { Literatura dramática y teatro }\end{array}$ \\
\hline $\begin{array}{l}\text { EXPRESION VISUALTransformación de mensajes y/o necesidades socia- } \\
\text { les en imágenes, dibujos, modelos tridimensionales y en general en obras } \\
\text { creativas visuales. }\end{array}$ & $\begin{array}{l}\text { Arquitecto } \\
\text { Arquitectura del paisaje } \\
\text { Artes visuales } \\
\text { Comunicación gráfica } \\
\text { Diseño gráfico } \\
\text { Diseño industrial } \\
\text { Urbanismo }\end{array}$ \\
\hline $\begin{array}{l}\text { CANTO Y MUSICA } \\
\text { Todas estas carreras están dedicadas al estudio de la música, la interpre- } \\
\text { tación y/o creación musical. }\end{array}$ & $\begin{array}{l}\text { Licenciado en canto } \\
\text { Licenciado en composición } \\
\text { Licenciado en educación musical } \\
\text { Licenciado en etnomusicología } \\
\text { Licenciado instrumentista } \\
\text { Licenciado en piano }\end{array}$ \\
\hline
\end{tabular}

